\title{
TODA LA VERDAD Y NADA MÁS QUE LA VERDAD*
}

\author{
Susan Haack ** \\ Universidad de Miami
}

RESUMEN. «Muchas verdades son pronunciadas, para que más puedan ser ocultadas», escribió el magistrado Darling en 1879. Empezando con una articulación de la distinción entre verdad (el fenómeno o concepto) y las verdades (proposiciones verdaderas particulares), este trabajo está en gran parte dedicado a: 1) desarrollar una explicación del doble significado de "parcialmente verdadero» - «verdad-en-parte» vs. «parte de la verdad»-, y 2) entender las razones, y los peligros, de la tendencia muy común a decir sólo parte de la verdad relevante. gaño.

Palabras clave: verdad, verdades, verdad parcial, RAMSEY, laconismo, vaguedad, en-

ABSTRACT. «Much truth is spoken, that more may be concealed», wrote Mr. Justice Darling in 1879. Opening with an articulation of the distinction between truth (the phenomenon or concept) and truths (particular true propositions), this paper is largely devoted to: 1) developing an account of the dual meaning of «partially true» — «true-in-part» vs. «part of the truth»-; and 2) understanding the reasons for, and the dangers of, the very common tendency to tell only part of the relevant truth.

Keywords: truth, truths, partial truth, RAMSEY, laconicism, vagueness, deception.

\footnotetext{
* Fecha de recepción: 2 de abril de 2012. Fecha de aceptación: 7 de mayo de 2012. 2008.

Título original: «The Whole Truth and Nothing but the Truth», en Midwest Studies in Philosophy, XXXII,

(c) S. HaAcK, 2008. Todos los derechos reservados. Traducción al español de A. SANTACOlOMA SANTACOLOMA con la asistencia de C. VÁzQUEZ Rojas.

** S. HAACK, Distinguished Professor in the Humanities, Cooper Senior Scholar en Artes y Ciencias, profesora de filosofía y de derecho de la Universidad de Miami, shaack@law.miami.edu.
} 


\section{«Much truth is spoken, that more may be concealed».}

Mr. Justice Darling (1879) ${ }^{1}$

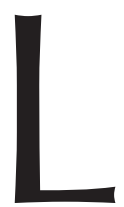

a palabra inglesa «truth» (como la palabra en castellano, «verdad») es utilizada algunas veces como un nombre abstracto: cuando se usa así, se está haciendo referencia al concepto de verdad o, como algunos prefieren decir, a la propiedad de ser verdadero, o al significado de «truth» y sus sinónimos en otros idiomas. También es utilizada para referirse a las cosas abarcadas en la extensión del concepto, es decir, proposiciones verdaderas, creencias verdaderas, afirmaciones verdaderas, teorías verdaderas, etc. ${ }^{2}$. En inglés sólo hay una palabra que hace ambos trabajos; la distinción en los usos sería más evidente si para el primero tuviéramos la palabra «true-ness» (cualidad de ser verdadero). Pero hay una indicación gramatical de la diferencia entre los dos usos: el segundo, mas no el primero, acepta un artículo indefinido (como en la oración de apertura de Pride and Prejudice: «It is a truth universally acknowledged that a single man in possession of a good fortune must be in want of a wife») y la forma plural (por ejemplo, cuando hablamos de «the truths of arithmetic» o en la segunda oración de la Declaración de Independencia Americana: «We hold these truths to be self-evident, that all men are created equal...») ${ }^{3}$. La palabra «falsehood» (falsedad) funciona de forma similar, sirviendo también como un sustantivo abstracto que refiere al concepto o a la propiedad de «falseness» (cualidad de ser falso) y como un sustantivo común para referir a proposiciones falsas, etc.; y, al igual que «verdad», en el segundo uso, la palabra acepta la forma plural y, aunque es más inusual, el artículo indefinido. «Falsity» (falsedad), sin embargo, parece funcionar, salvo en raras excepciones, como un sustantivo abstracto.

Este doble rol de las palabras «truth» $\mathrm{y}$ «verdad» puede generar problemas. Algunos, confundiendo los dos usos, tratan al sustantivo abstracto «verdad» como si se refiriese a algún tipo de proposición verdadera muy especial e indispensable; y, por ello, hablan con reverencia de la Verdad, usando una «V» mayúscula, como en «la Verdad os hará libres». Otros, considerando perturbadora este tipo de reverencia por «la Verdad», llegaron confusamente a la conclusión equivocada de que es una mera superstición asignar cualquier valor a la verdad o, incluso, que debemos rechazar hablar sobre la verdad. Por ejemplo, P. CHURCHLAND escribe que «la verdad, sea esta lo que sea, definitivamente es lo postrero» ${ }^{4}$; S. HARDING que «la verdad — isea esta lo que

1 «Muchas verdades son pronunciadas, para que más puedan ser ocultadas». Ch. J. DARLING, Scintillae Juris, 3. ${ }^{a}$ ed., ampliada, London, Davis and Son, 1879, 73.

2 Existe una dualidad similar con la palabra «derecho»: compárese «El derecho es la voluntad del soberano» y «El derecho en Michigan fue modificado en el 2004 respecto a la admisibilidad de las pruebas científicas». Vid. la cita 18 más adelante.

3 Respectivamente: «Es una verdad universalmente reconocida que un hombre soltero en posesión de una buena fortuna debe estar en necesidad de una esposa». J. Austen, «Pride and Prejudice» (1813), en The Works of Jane Austen, London, Spring Books, 1966, 171. Existe traducción al castellano, Orgullo y Prejuicio, Madrid, Edaf Narrativa, 2002. «Las verdades de la aritmética». Y: «Nosotros tenemos estas verdades como auto-evidentes: que todos los hombres son creados iguales...».

4 P. Smith ChURCHLAND, «Epistemology in the Age of Neuroscience», Journal of Philosophy, 75, núm. 10, $1987,544-553,549$. 
sea! - no os hará libres» ${ }^{5}$; J. HEAL que «la verdad es generalmente concebida como algo bueno», sin embargo este «aparente truismo» es erróneo porque «verdad» no es en absoluto un término evaluativo ${ }^{6}$; S. STICH que «una vez que tengamos una visión clara sobre la cuestión, la mayoría de nosotros no encontraremos ningún valor... en tener creencias verdaderas» ${ }^{7}$, y R. RORTY que él «no encuentra utilidad para nociones como "verdad objetiva" ${ }^{8}$.

He hecho ya un análisis de estos dos tipos de confusión en otros trabajos ${ }^{9}$; aquí, quiero concentrarme en un tercer tipo de confusión, también provocado por los dos usos de la palabra «truth» (o «verdad»); atribuyéndosele a verdad, entendida como veracidad, propiedades de algunas, pero no todas, las verdades ${ }^{10}$. Existe una verdad, una veracidad o un concepto de verdad. Pero hay muchas verdades, es decir, muchas y varias proposiciones, etc., verdaderas. La verdad no depende de lo que nosotros creemos o aceptamos, no es relativa a la cultura, a la comunidad, a una teoría o a un individuo; y no es una cuestión gradual, ni una conglomeración de propiedades que pueden estar total o parcialmente satisfechas. Sin embargo, algunas verdades son tales en virtud de algo que nosotros hacemos; otras en virtud de lo que creemos; y algunas verdades sólo tienen sentido relativizadas a un tiempo, a un lugar o a una cultura. Por otra parte, algunas proposiciones verdaderas son en algún grado vagas, otras son más precisas; algunas son, en una u otra forma, parciales y otras más completas. La verdad, en pocas palabras, es simple; las verdades, en cambio, no lo son. Las verdades vienen en todo tipo de formas y tamaños; y la mayor parte del tiempo transitamos en el casi, en lo aproximado o en lo parcialmente verdadero.

El propósito del primer apartado de este trabajo es articular estos contrastes con mayor cuidado; y, el del segundo es explorar algunas dimensiones epistemológicas, retóricas y prácticas de la verdad parcial.

\section{PROBLEMAS CON «VERDAD» $\mathrm{Y}$ «VERDADES»}

No desconozco que «truth» se usa en inglés (al igual que «verdadero» en castellano) como un nombre singular abstracto no sólo de creencias, teorías, afirmaciones,

\footnotetext{
5 S. HaRDING, Whose Science? Whose Knowledge?, Ithaca, NY, Cornell University Press, 1991, xi.

6 J. HEaL, «The Desinterested Search for Truth», Proceedings of the Aristotelian Society, 88, 1987-1988, 97-108, 97.

7 S. P. STich, The Fragmentation of Reason: Preface to a Pragmatic Theory of Cognitive Evaluation, Cambridge, MA, Bradford Books, MIT Press, 1990, 101.

8 R. RORTY, Essays on Heidegger and Others, Cambridge, England, Cambridge University Press, 1991, 86.

9 Vid. S. HAACK, Evidence and Inquiry: Towards Reconstruction in Epistemology, Oxford, Blackwell, 1993; 2. ${ }^{a}$ ed., Evidence and Inquiry: A Pragmatist Reconstruction of Epistemology, Amherst, NY, Prometheus Books, 2009, capítulos 8 y 9; «Confessions of an Old-Fashioned Prig», Manifesto of a Passionate Moderate: Unfashionable Essays, Chicago, University of Chicago Press, 1998, 7-30; «Staying for an Answer: The Untidy Process of Groping for Truth», Times Literary Supplement, 9 de julio de 1999, 12-14, reimpreso en S. HAACK, Putting Philosophy to Work: Inquiry and Its Place in Culture, Amherst, NY, Prometheus Books, 2008, 25-36; «Engaging with the Engaged Inquirer: Response to Mark Migotti», en C. DE WAAL (ed.), Susan Haack, A Lady of Distinctions, Amherst, NY, Prometheus Books, 2007, 277-280.

${ }_{10}$ Esto ya estaba implícito en uno de mis trabajos anteriores, «The Unity of Truth and the Plurality of Truths», Principia 9.1-2, 2005, 87-110; reimpreso en S. HAACK, Putting Philosophy to Work, vid. cita 9, 43-60. El intercambio de algunos correos con S. PETHICK me ayudó a ponerlo explícito.
} 
declaraciones, proposiciones, etc., sino también de personas, imágenes, etc., que no son proposicionales. Hablamos de un «verdadero amigo», una «verdadera semejanza», un «verdadero estudioso», un «verdadero amor»; describimos a la ballena como un «verdadero mamífero» y no como un «verdadero pez»; el título memorable de una película habla inclusive de True Grit (literalmente «verdadera persistencia», pero en castellano la película fue intitulada Valor de Ley). Estos usos no proposicionales están estrechamente relacionados con los proposicionales, ya que comunican que algo es «real, genuino, verdaderamente (un) F»: verdaderamente un amigo, verdaderamente una semejanza, verdaderamente un mamífero, verdaderamente un estudioso, verdaderamente persistente, etc. En otros usos, «verdadero» no es fácilmente asimilable a la verdad de las proposiciones; por ejemplo, cuando hablamos de «being true to oneself» para significar algo como «comportarse acorde con las creencias y el carácter de uno mismo», o cuando describimos a una viga o a una juntura como «out of true» (fuera de alineación), queriendo decir que está torcida, inclinada o ladeada. Pero en adelante, me limitaré a usar «verdadero» como un predicado aplicable a proposiciones, afirmaciones, creencias y similares.

Doy por sentado que verdad entendida como «true-ness» (ser verdadero), no puede ser identificada con la aceptación de algo como verdadero; pues lo que es aceptado como verdadero puede no ser verdadero y lo que es verdadero puede no ser aceptado como tal. Tampoco puede identificarse verdad con creencia, ni siquiera con creencia justificada; pues lo que se cree, inclusive si la creencia tiene fundamento en pruebas sólidas, puede no ser verdadero y lo que es verdadero puede que no se crea. Tampoco puede identificarse verdad con conocimiento; pues mientras lo que se conoce debe ser verdadero, lo que es verdadero puede no ser conocido. Tampoco se puede identificar verdad con acuerdo; pues si acordamos que $p$, acordamos que es verdadero que $p$, podríamos acordar que $p$ cuando no es verdadero que $p$ y podríamos no acordar que $p$ cuando es verdadero que $p$. Y tampoco se puede identificar verdad con sinceridad o candor, pues aunque una persona sincera esté dispuesta a hablar de lo que ella cree que es verdad, si su creencia es falsa, su afirmación, aunque sincera, será falsa.

También doy por sentado que entre la multiplicidad de teorías filosóficas sobre la verdad, las más plausibles son, en intento o en efecto, generalizaciones de la intuición aristotélica «decir de lo que no es que no es o de lo que es que es, es verdadero» ${ }^{11}$. Algunas de éstas, diversas variantes de la teoría de la correspondencia, cambiaron aquellos adverbios enfáticos con los que se llegó a decir que $p$ es verdadero sólo en el caso de que realmente, de hecho, $p$ en las complejidades metafísicas; interpretando la verdad como una relación, estructural o convencional, de proposiciones o afirmaciones con los hechos o con la realidad. Otras, como la teoría semántica de A. TARSKI, la teoría laconicista de F. RAMSEY y las muchas y variadas teorías contemporáneas descendientes de éstas, como las deflacionistas, las minimalistas, las decitacionalistas, las pronunciativas, etc., no exigen tales inversiones ontológicas tan grandes. Pero el mismo TARSKI dudó en la aplicación de su teoría fuera del dominio de los lenguajes reglados y formalizados de la lógica y las matemáticas; «la mera posibilidad de un uso consistente de

11 «To say of what is not that it is not, or of what is that it is, is true». ARISTOTLE, Metaphysics, trans. W. D. Ross, Book Gamma (IV), 7, 1011b25, en The Basic Works of Aristotle, R. McKeON (ed.), New York, Random House, 1941, 749. 
la expresión "enunciado verdadero" que esté en armonía con las leyes de la lógica y el espíritu del lenguaje cotidiano», escribió, «parece ser muy cuestionable» ${ }^{12}$. Y ahora que el polvo del «Programa de Davidson» se ha asentado ${ }^{13}$, podemos ver que las reservas de TARSKI eran proféticas. El enfoque más prometedor parece ser algo cercano a la simple afirmación de RAMSEY: «La creencia que $p .$. es verdadera si y sólo si $p$; por ejemplo, la creencia que Smith es o un mentiroso o un tonto es verdadera si Smith es un mentiroso o un tonto y no de otro modo» ${ }^{14}$.

Aunque fue ampliamente conocida como «teoría de la redundancia» de la verdad, la teoría de RAMSEY, lacónica como es, no supone que «verdadero» no tenga un genuino rol que jugar; RAMSEY era muy consciente de que mientras «es verdadero que» es eliminable en atribuciones directas de verdad (como cuando decimos que es verdadero que Julio César cruzó el Rubicón), tiene un rol sustancial en las atribuciones indirectas de verdad (por ejemplo cuando decimos que PLATÓn dijo algunas cosas verdaderas y otras falsas). RAMSEY estaba también muy consciente de que su teoría dejaba muchas cuestiones aún por responder — preguntas técnicas sobre los cuantificadores necesarios para explicar esas atribuciones indirectas, así como cuestiones filosóficas sobre la representación (qué es esto para ser la proposición que $p$ ) y la realidad (qué significa «ser el caso que $p »)$ - Y Y él no pretende ofrecer un criterio de verdad, sino que reconoce que su teoría deja cuestiones epistemológicas sin tocar.

Pero la simple formulación inicial de RAMSEY basta para articular lo que quiero afirmar:

- Que aunque hay muchas y variadas proposiciones verdaderas, sólo hay una verdad.

- Que aunque algunas proposiciones verdaderas versan sobre cosas que dependen de nosotros, la verdad es objetiva.

- Que aunque algunas proposiciones cobran sentido sólo si se entienden como relativas a un sitio, tiempo, cultura, sistema jurídico, etc., la verdad no es relativa.

- Que aunque algunas proposiciones verdaderas son vagas, la verdad no es gradual.

- Que aunque algunas proposiciones sólo son parcialmente verdaderas, la verdad no se descompone en partes.

El principio básico que dice que una proposición es verdadera sólo en caso que sea la proposición que $p$, y $p$, es aplicable con independencia del tipo de proposición de que se trate (asumiendo que es susceptible de ser verdadera), bien sea un teorema matemático o una conjetura histórica, la profecía de un inminente desastre o una predicción meteorológica, una proposición sobre literatura o una proposición sobre el derecho, una teoría de la física o una generalización sociológica, un enunciado sobre

12 A. TARSKI, «The Concept of Truth in Formalized Languages» (publicado primero en polaco en 1933), en Logic, Semantics, Metamathematics, trad. de J. H. WoOdgER, Oxford, Clarendon Press, 1956, 152-278, 165.

${ }_{13}$ En «A Nice Derangement of Epitaphs», en E. LEPORE (ed.), Truth and Interpretation, Oxford, Blackwell, 1986, 433-446, el mismo D. DAVIDSON efectivamente reconoce que su proyecto de dar una teoría tarskiana de significados para los lenguajes naturales ha sido erróneamente concebido, escribiendo (445-446) que él ha llegado a la conclusión que «no hay cosa tal como el lenguaje, no si el lenguaje es alguna cosa como lo que muchos filósofos han supuesto».

${ }^{14}$ F. P. RAmSey, On Truth (escritos de 1927-1929), N. ResCher y U. Majer (eds.), Dordrecht, The Netherlands, Kluwer, 1992, 12. 
lo que desayuné el 11 de septiembre del 2001 o una predicción sobre quién ganará las próximas elecciones presidenciales o (asumiendo que las proposiciones de la ética son susceptibles de verdad o falsedad) una proposición sobre las acciones, los motivos, los rasgos de carácter de las personas, las reglas, los acuerdos, las instituciones sociales, etc., que son moralmente deseables o indeseables ${ }^{15}$. En una versión más simple, cuando digo que aunque hay muchas verdades, muchas teorías sobre la verdad y muchas concepciones de la verdad, algunas correctas y otras no, sólo hay una verdad: lo que quiere decir cuando afirmamos que un enunciado es verdadero es lo mismo, sin importar de qué trate este enunciado.

La misma simple fórmula, una proposición es verdadera sólo en caso que sea la proposición que $p$, $y p$, es suficiente para decirnos que si una proposición es verdadera o es falsa, es una cuestión objetiva; es decir, para que una proposición sea verdadera no es necesario ni tampoco suficiente que usted o yo o alguien la crea. Esto es bastante obvio cuando hablamos de afirmaciones y teorías sobre fenómenos y eventos naturales que no son producto de nuestras construcciones. Pero no es menos cierto cuando se trata de afirmaciones y teorías sobre fenómenos y eventos que solamente ocurren en circunstancias artificiales creadas en el laboratorio, aun cuando éstas son creaciones nuestras; porque la verdad o la falsedad de tales proposiciones es independiente de si usted o yo o quien sea cree que son verdaderas o falsas. Tampoco es menos cierto respecto a las verdades jurídicas, aunque éstas sean verdaderas por creación legislativa o porque tienen origen en precedentes, es decir, en cosas que la gente hace; o respecto a proposiciones o teorías científico-sociales, aunque sean acerca de fenómenos sociales e instituciones constituidas en parte por las creencias, las esperanzas, los temores, etc., de las personas. Consideremos, por ejemplo, la proposición enunciada por G. ORWELL, «la clase trabajadora inglesa... "está marcada en la lengua" ${ }^{16}$, lo que significa en otras palabras, que en Inglaterra la clase social está estrechamente relacionada con el acento. Si esto es verdadero o falso, está estrechamente relacionado con si una proporción suficiente de personas en Inglaterra lo creen; su valor de verdad es, sin embargo, independiente de que usted o yo o cualquier persona crea que esto es verdadero o que es falso.

Algunas proposiciones son incompletas y, por ende, no son susceptibles de verdad o falsedad, a menos que sean entendidas como restringidas a un lugar, a un tiempo o a una cultura (algunos prefieren interpretar tales supuestas proposiciones sólo como funciones proposicionales que necesitan ser completadas antes de tener el estatus de proposición). Una proposición que dice que la ley es de una forma u otra, por ejemplo, sólo tiene sentido cuando se interpreta como relativa a un sistema jurídico y en un tiempo determinado. Alguna vez fue verdad, pero ya no lo es, que en el derecho inglés el castigo por robarse una oveja era la muerte ${ }^{17}$; desde 1952 es verdad que en

15 Y en forma similar, mutatis mutandis, para proposiciones de la estética (y para cualquier otra clase de proposiciones donde la cuestión de si son susceptibles de verdad o falsedad está en disputa).

16 Vid. G. ORwell, In Front of Your Nose: The Collected Essays, Journalism and Letters of George Orwell, vol. IV, S. Orwell e I. ANGus (eds.), New York, Harcourt, Brace and World, 1968, 5. Él se está refiriendo a W. LEWIS, The Vulgar Streak, Santa Barbara, CA, Black Sparrow Press, 1985, 38, donde MARTIN habla de «la superstición de la clase como una gran soga alrededor del cuello de alguien —en el cual mi lengua fue bien marcada $\longrightarrow$.

17 La ley que establecía que una persona detenida por robar una oveja debía ser condenada a muerte, se aprobó «en los años séptimo y octavo de reinado del rey George IV» (esta, por supuesto, era la realidad 
el derecho del estado de Florida la admisibilidad de las pruebas científicas está sujeta al criterio Frye y esto fue verdad también en el estado de Michigan desde 1956 hasta el 2004 (cuando el ordenamiento respectivo fue reformado) ${ }^{18}$. Muchas proposiciones científico-sociales, similarmente, deben ser entendidas como relativas a un lugar y a un tiempo. En el tiempo en el que yo crecí en Inglaterra, era verdad que la clase social estaba relacionada estrechamente con el acento; sin embargo, a juzgar por el discurso de los presentadores de noticias y de los actores de los programas de televisión británicos que veo hoy en día en Estados Unidos, esto ya no es verdadero. Pero si es verdadero o si es falso que en el derecho inglés de 1831 la pena por robarse una oveja era la muerte o, si es verdadero o falso que en el 2008 en Inglaterra la clase y el acento están estrechamente relacionados, no es relativo ni al tiempo ni al lugar. Que algunas verdades sean relativas a un lugar, a un tiempo, a una cultura, a un sistema jurídico, etc., no implica que la verdad sea relativa.

Algunas verdades son vagas, en diversas maneras y en diversos grados. «En diversos grados» pretende indicar que no se trata de que algunas verdades sean vagas y otras precisas, sino que algunas son más vagas y otras más precisas; la frase «él es alto» es más vaga que, por ejemplo, «él es significativamente más alto que el hombre japonés promedio», pero ésta es más vaga que «él mide 2,01168 m». Para comunicar la real complejidad del asunto, sin embargo, se requeriría más que estos ejemplos simples, por esto decimos que un idioma es realmente un racimo de idiolectos, un vasto y denso enlace de patrones bastante similares de uso — casi-iguales pero no idénticos. Y todo lenguaje natural está en constante movimiento y cambiando un poco aquí y allá. Algunas palabras y frases están regladas, se vuelven menos vagas, cuando son adoptadas como herramientas especializadas en un área u otra y, en cambio, otras pierden la especificidad de su significado, se vuelven más vagas cuando se tornan más comunes en los discursos populares o en la jerga propagandística ${ }^{19}$.

«En diversas maneras» pretende indicar que existen muchas fuentes de vaguedad. Los entusiastas de la lógica difusa, y no pocos filósofos, han estado preocupados con la vaguedad de predicados como «alto», «máximo», «calvo», «ruidoso», «confiable», etc., que expresan propiedades que se presentan en grados. Pero otros tipos de expresiones, además de los predicados, pueden ser graduales: sustantivos como «cúmulo», «multitud» o «montón», por ejemplo, o adverbios como «rápidamente», «justamente», «razonablemente», o «normalmente». Existen además frases que resultan vagas para cuantificar como «unos pocos» o «muchos» - lista a la que C. S. PEIRCE habría

jurídica que está tras el refrán, «bien podría ser colgado como un cordero por una oveja»). La ley fue derogada en 1832. Estatutos del Reino Unido de Gran Bretaña e Irlanda, 2 y 3 William IV, 1832, cap. LXIII, s. I (11 de Julio de 1832).

18 De acuerdo con el criterio Frye [emitido en el caso Frye v. United States, 54 App.DC 46, 293, F. 1013 (1923)], las pruebas científicas noveles sólo serían admisibles si el principio en que se basan está «suficientemente establecido para ser aceptado generalmente en el área de la ciencia a la que éste pertenece». Florida adoptó este criterio en el caso Kaminski vs. State 63 So.3d 339 (1952) y continúa aceptándolo; Michigan adoptó este criterio en el caso People vs. Davis, 34 Mich.348 (1956), pero lo abandonó en 2004, cuando las nuevas reglas de prueba de Michigan adoptaron el estándar Daubert [derivado del caso Daubert vs. Merrell Dow Pharmaceuticals, Inc., 509 U.S. 579, 113 (1993 S. Ct. 2786)], de acuerdo con el cual la prueba pericial, científica o no, sólo es admisible si es relevante y fiable.

19 Vid. S. HAACK, «The Growth of Meaning and the Limits of Formalism: In Science, in Law» (2007), Análisis Filosófico, XXIX.1, 2009, 5-29. 
añadido el cuantificador de existencia «alguno» ${ }^{20}$ (lo que me recuerda mencionar que B. RuSSELl alguna vez dijo que las descripciones indefinidas, por ejemplo «un hombre» en «conocí a un hombre»o «vi a un unicornio», son ambiguas) ${ }^{21}-$. Y hay otras formas de indeterminación del significado además de la gradación. Palabras que pueden ser familiares en una conversación como «agradable» o «bien», así como palabras más efímeras o de moda, como «binky» o «funky»—que, según J. BARZUM, «definidas por un experto significan "muy bien o hermoso, sólido, barato, oloroso o, en general, no-bueno"»— deben su utilidad, en parte, precisamente a su significado parasitario, a su carencia de sentido específico; dependen, como señala BARZUM, «del tono, de la mirada, o del movimiento de la ceja» ${ }^{22}$. Y luego, están las palabras que BARZUM llama «escurridiza jerga de las relaciones públicas» ${ }^{23}$ que, en parte, deben su utilidad al hecho de que han sido casi completamente despojadas de sus significados, aun cuando conservan sus connotaciones favorables.

Esta «escurridiza jerga de las relaciones publicas» hoy en día está por todos lados, no sólo en el discurso político («progresista», «cambio», «democrático»), en la publicidad («nuevo», «mejorado», «científico») y en la jerga de los vendedores de bienes raíces («cocina gourmet», «Gran Sala»), sino que se ha convertido en el pan de cada día de todo tipo de marketing y de la academia («prestigioso», «excelencia», «distinguido») ${ }^{24}$.

Podría parecer que la ambigüedad es un asunto de las expresiones que tienen muchos significados y, por ello, sería todo lo opuesto a la vaguedad, la cual es un asunto de las expresiones que no tienen significado determinado. Pero cuando se usan términos que tienen varios o muchos significados sin especificar su sentido, el resultado es a menudo difícil de distinguir de la vaguedad. Los términos técnicos en filosofía frecuentemente sufren esta suerte; por ejemplo «realismo» ${ }^{25}$, «naturalismo» ${ }^{26}$, «epistemología social» y «epistemología de las virtudes» ${ }^{27}$, entre muchos otros. Hay además un tipo peculiar de vaguedad que puede ser descrito como «pseudo-precisión», en el cual los términos centrales de un significado no muy determinado son disfrazados con formalismos lógicos o matemáticos para dar la impresión de que se está siendo riguroso —un

20 C. S. Peirce, Collected Papers, Ch. Hartshorne, P. Weiss (vols. 7 y 8) y A. Burks (eds.), Cambridge, Harvard University Press, 1931-1958, 5.446, 1905 (las referencias a los Collected Papers se encuentran por volumen y número de párrafo).

21 B. Russell, Introduction to Mathematical Philosophy, London, Allen and Unwin, 1919, capítulo XVI.

22 J. BARZum, «What Are Mistakes and Why», en BARZum, A Word or Two Before You Go, Middletown, CT, Wesleyan University Press, 1986, 3-9, 8. Compárese estos usos de «funky», con los que me encontré mientras escribía este artículo: «La cabaña de Chris era funky y encantadora» (J. PATTERSON, $1^{\text {st }}$ to Die, New York, Warner Books, 2001, 370); «...una tendencia muy fuerte que llegó y se fue rápidamente en la décadas funky de 1960 y 1970, cuando las personas que sabían más de moda salieron con la idea de colores psicodélicos» (R. Dodes y Ch. Passariello, «Gasp! Polyester is the New Name in Paris Fashion», Wall Street Journal, 1 de marzo de 2008, A.1).

23 J. Barzum, A Stroll With William James (1983), Chicago, University of Chicago Press, 1984, 223.

24 Vid. S. HAACK, «Preposterism and Its Consequences», Manifesto of a Passionate Moderate: Unfashionable Essays, Chicago, University of Chicago Press, 1984, 223.

${ }_{25}$ Sobre los muchos y cambiantes significados de «realismo», vid. S. HAACK, «Realisms and Their Rivals: Recovering Our Innocence», Facta Philosophica, 4, 1, 2002, 67-88.

26 Sobre los muchos y cambiantes significados de «naturalismo», vid. S. HAACK, Evidence and Inquiry (vid. cita 9), capítulo 6.

27 Sobre los muchos y cambiantes significados de «epistemología social» y «epistemología de las virtudes», vid. S. HAACK, Evidence and Inquiry, prologo de la segunda edición (cita 9). 
tipo de vaguedad sumamente común en las ciencias sociales pero, en mi opinión, no menos ubicua en la filosofía neo-analítica-.

La vaguedad normalmente se trata como algo opuesto a la precisión; y la precisión, como la vaguedad, es más sutil de lo que a veces se supone. Tal vez, en lo primero que pensamos es en la precisión medible, cuantificable, en una ordenación sintácticamente expresable que es valorada correctamente por matemáticos, científicos y lógicos, donde los términos graduales son reemplazados por sustitutos que están definidos de forma precisa. Pero no debemos olvidarnos de otro tipo de exactitud correctamente valorada no sólo por poetas, dramaturgos y novelistas, no sólo por el ingenioso y el devoto de la palabra exacta, sino por todo aquel que respeta la prosa eficaz. Como ORWELL nos recuerda en La política y el idioma inglés, «sobre todo, es necesario» para escribir bien «dejar que el sentido elija las palabras y no al contrario» ${ }^{28}$ : este es el tipo de exactitud «poética» que se logra al elegir la palabra o la frase que resulta menos familiar pero más individualizada, en lugar de elegir de forma indolente a las que resultan más comunes, o plantear una nueva metáfora o similitud, en lugar de recurrir a lo viejo y estereotipado.

Algunas veces se piensa que la vaguedad es un obstáculo para la verdad; no obstante, es al contrario: en realidad es más fácil decir algo verdadero si no se es demasiado preciso: compare «él es bastante alto» con «él mide 1,88976 metros con sus calcetines más gruesos», o «New York tiene una extensa población» con «el Estado de New York tiene una población de $n$ adultos y $m$ niños menores de 18 años». Calificar una afirmación como «accurate» («acertada»), entiendo yo, es decir que es «precise» («exacta») y también que es «true» («verdadera») [esto explica por qué en inglés podemos describir una afirmación como «precisely wrong» o «exactly wrong» pero no como «accurately wrong» («precisamente» o «atinadamente» incorrecta, pero no como «acertadamente» incorrecta)].

Pero aunque muchas verdades son vagas, la verdad, o «true-ness» (veracidad), no es una cuestión gradual. Una forma de sustentar esto sería, de nuevo, apelando a la fórmula de RAMSEY. Otra forma sería señalando ciertos tipos de pruebas lingüísticas (aunque esto tiene la desventaja de ser un tanto local; idealmente, se necesitaría explorar el fenómeno correspondiente en varios idiomas, preferiblemente no cercanos entre ellos). Aun así, por lo menos en el inglés, las pruebas son muy persuasivas. Los tipos de modificadores adverbiales que usamos rutinariamente con predicados como «tall» («alto»), «intelligent» («inteligente») o «rich» («rico»), que expresan propiedades que se presentan en grados — modificadores como «fairly» («bastante»), «rather» («un poco»), «extremely» («extremadamente»), «unusually» («inusualmente»), «abnormally» («anormalmente»)— no pueden ser usados para modificar «true». Por supuesto, algunas veces decimos que una afirmación es «quite true»; pero en este uso «quite» debe entenderse en un sentido equivalente a «absolutamente» o «perfectamente» y no en el sentido (británico) equivalente a «suficiente»o «un poco». Y algunas veces decimos que una afirmación es «very true»; pero esto no significa que esta sea verdadera en un grado superior sino que no sólo es verdadera sino muy im-

28 G. ORWELL, «Politics and the English Language», en S. ORWELL e I. ANGus (eds.), In Front of Your Nose: The Collected Essays, Journalism, and Letters of George Orwell, vol. IV, New York, Harcourt, Brace and World, 1968, 127-140, 129. Vid. también BARZum, A Word or Two Before you Go (cita 22). 
portante para el punto que trata. A veces, también calificamos a ciertas afirmaciones como «approximately true» («aproximadamente» verdadera) o «roughly true» («más o menos» verdadera), o, más idiomáticamente, «roughly right». Pero estas locuciones no prueban que la verdad es una cuestión gradual sino que las afirmaciones verdaderas pueden ser más o menos vagas. «" $P$ ” es aproximadamente verdadero» es una locución útil pero prescindible en favor de una más transparente: «"aproximadamente $p$ " es verdadero» ${ }^{29}$.

Otra vez, algunas proposiciones, afirmaciones, etc., son, como decimos, «no completamente verdaderas» sino sólo «parcialmente» o «medianamente» verdaderas (no creo haber leído u oído nunca «verdadero en un tercio» o «verdadero en un cuarto»; aunque sí encontré que J. FEKETE escribió «verdad fraccional» en una sección de su libro Moral Panic titulada «iPor favor, sólo verdades a medias!») ${ }^{30}$. Describir a una afirmación como parcialmente verdadera puede significar dos cosas muy diferentes: i) que es verdadera en parte y, a la vez, falsa en parte; o, ii) que es verdadera pero incompleta. Una afirmación que es parcialmente verdadera en el sentido i) (es decir, verdadera sólo en parte) será también parcialmente verdadera en el sentido ii) (es decir, no será toda la verdad); sin embargo, la implicación contraria no se sostiene. Una afirmación que es parcialmente verdadera en el primer sentido es insatisfactoria porque no es nada excepto la verdad; una declaración parcialmente verdadera en el segundo sentido es insatisfactoria porque no es toda la verdad.

El primer significado de «parcialmente verdadero», «parte de " $p$ " es verdadero», es relativamente sencillo. En el caso más simple, una conjunción (o algunos de los enunciados conjuntos) en un enunciado conjuntivo es (o son) verdadera(s) y otra (u otras) falsa(s); por ejemplo, «ella era pobre pero honesta» es en parte verdadera si ella era pobre pero no era honesta o si era honesta pero no pobre. En casos un poco menos simples, el carácter conjuntivo del enunciado no es tan evidente, pero está implícito en la conjunción de predicados, por ejemplo, «él es un erudito y un caballero» es en parte verdadera si él es un erudito pero no es un caballero o si es un caballero pero no es un erudito. En otros casos, el carácter conjuntivo de un enunciado es menos explícito; está implícito en una frase adverbial: por ejemplo, podríamos decir que «lo vi por última vez el día de Navidad en 1974» es verdadera en parte si yo le vi por última vez en 1974 pero no era el día de Navidad sino el día de Nochebuena, o si la última vez que lo vi fue el día de Navidad pero en 1975 y no en 1974, etc. (como sugerí en mi libro Deviant Logic, la verdad parcial en este sentido podría representarse con una lógica multivalente que satisfaga las matrices no-estándar de Emil POST) ${ }^{31}$.

${ }^{29}$ Hay un análisis más detallado de las pruebas lingüísticas en mi artículo «Is Truth Flat or Bumpy?», en D. H. MelloR (ed.), Prospects for Pragmatism, Cambridge, Cambridge University Press, 1980, 1-20; reimpreso en S. HAACK, Deviant Logic, Fuzzy Logic: Beyond the Formalism, Chicago, University of Chicago Press, 1996, 243-258. Existe traducción al castellano de la primera edición: 1980, Lógica Divergente, Madrid, Paraninfo, S. A. Ediciones. Puede verse también S. HAACK, «Do We Need "Fuzzy Logic"?», International Journal of ManMachine Studies, 11, 1979, 432-445, también reimpreso en Deviant Logic, Fuzzy Logic, 232-242.

${ }^{30}$ J. Fekete, Moral Panic: Biopolitics Rising, Montreal, Robert Davies Publishing, 1994; 2. ed., revisada, 1995, 97. Ahora me he dado cuenta de que estaba equivocada cuando afirmé que nunca había leído «verdadero en un cuarto». Sí lo hice, en la novela de S. LEwIS, Arrowsmith: cuando el Dr. Max Gottlieb le dice a Martin Arrowsmith que «el científico es... tan religioso que no aceptará un cuarto de verdad porque son un insulto a su fe». S. LewIS, Arrowsmith, 1927, New York, Signet Classics, 1961, 1998, 278.

31 Vid. S. HAACK, Deviant Logic (cita 29), 62-63, las mismas páginas en ambas ediciones. 
El segundo significado posible de « " $p$ " es parcialmente verdadero», «" $p$ " es parte de la verdad», es a la vez significativamente menos sencillo y significativamente más interesante. Después de todo, ninguna afirmación, con independencia de lo comprehensiva que sea, puede representar toda la verdad acerca de absolutamente todo; en este sentido de «verdad parcial», toda verdad debe ser parcial. Usted puede estarse preguntando ¿qué pasa cuando le pedimos a un testigo que jure que el testimonio que rendirá será «la verdad, toda la verdad y nada más que la verdad»? Este es un asunto complicado; pero lo que estamos pidiendo, asumo, no es que el testigo deba decir toda la verdad acerca de todo, lo que es imposible, sino que debe decir la verdad como él cree que ésta es, sin omisiones relevantes. El punto queda más claro si consideramos cuando reclamamos a un político por no haber dicho toda la verdad sobre los costos que acarrearía poner en march a una propuesta o cuando nos quejamos porque el departamento de filosofía no dice toda la verdad sobre el grado de éxito en encontrar empleo para los estudiantes que completan con éxito su doctorado, etcétera.

La manera más obvia de decir menos que toda la verdad relevante es simplemente omitir información relevante, por ejemplo, jactarse de que $A$ obtuvo la titularidad en la Euphoric State ${ }^{32}$ y $B$ una posición de visitante en el Podunk College, pero omitiendo mencionar que varios doctorandos recién graduados no han conseguido ningún trabajo. Otra forma es usar términos clave en un sentido encubiertamente extendido. Una encuesta de 1993 reportó que en Canadá el 81 por 100 de las mujeres que estudiaban en las universidades y que tenían un noviazgo sufrieron de abusos sexuales; sin embargo, FEKETE argumenta que esta cifra alarmante es una de esas verdades fraccionales dado que el término «abuso sexual» fue usado en un sentido tan amplio que cubría casos desde haber sido violada después de ser amenazada con arma blanca o a punta de pistola, hasta el coqueteo no deseado ${ }^{33}$. Otra forma, también es el reconocimiento incompleto - una perturbadora y común forma de deshonestidad en los escritos académicos en filosofía y, sin lugar a dudas, en otras disciplinas- señalando que un pequeño elemento de lo que se está diciendo se deriva del trabajo de alguien más, mientras al mismo tiempo silenciosamente se toman «prestadas» las ideas de otro $\sin$ hacer el reconocimiento respectivo. Este furtivo truco retórico (del que he sido víctima más de una vez) puede ser descrito como «la condena del elogio débil»* *

Sin duda, la pregunta sobre qué omisiones constituyen una falla a la hora de decir toda la verdad (relevante), puede ser una cuestión tramposa. «Sobre qué» es un asunto vago y la relevancia es gradual. Que el testigo haya visto al acusado dispararle al fallecido, por ejemplo, es muy relevante para condenarlo; que el testigo haya visto al acusado en el vecindario alrededor de la hora del crimen es relevante pero en un grado mucho menor; etc. Además, si $p$ es relevante para $q$, y en qué grado, depende de los hechos. Si y en qué grado el hecho de que este medicamento cause cáncer a los animales es relevante para determinar si también causa cáncer en los seres humanos, por ejemplo,

32 D. Lodge, Changing Places: A Tale of Two Campuses (1975), Harmondsworth, Middlesex, Penguin Books, 1978.

33 J. FeKeTe, Moral Panic (cita 30), 60.

* N. del T.: La autora usa la expresión «condemnation by faint praise» que significa que alguien hace algún tipo de elogio que es tan débil o tan mínimo que resulta no exaltador de ninguna cualidad o mérito. En inglés la autora dice «or rather, by feint!'», haciendo un chiste verbal aprovechando la similitud en la pronunciación de $\ll$ faint $\gg($ elogio) y $« f e i n t »($ finta). 
depende de cuán similares sean fisiológicamente los animales en cuestión con los humanos (en los aspectos relevantes), de si las dosis administradas son comparables dado el tamaño de los animales y el del ser humano, etcétera.

Pero que algunas proposiciones sean sólo parcialmente verdaderas no implica que la verdad se descomponga en partes. Una verdad parcial en el primer sentido (una proposición que no es totalmente verdadera) es, estrictamente hablando, simplemente falsa. Y - a menos que sea también parcialmente verdadera en el primer sentido, es decir, sólo en parte verdadera - una verdad parcial en el segundo sentido (una proposición que no es toda la verdad relevante) es, estrictamente hablando, simplemente verdadera ${ }^{34}$.

\section{PROBLEMAS CON LA VERDAD PARCIAL (Y ALGUNOS CAPRICHOS CON LA VAGUEDAD)}

Estos argumentos arrojan cierta luz sobre preocupaciones respecto de la verdad. Cualquier descripción de un evento del pasado — una batalla, por ejemplo, la caída de un imperio, el nacimiento de una nación - será, inevitablemente, incompleta. La crónica de una batalla, por ejemplo, seguramente nos dirá quiénes ganaron, cuáles fueron las consecuencias de esa batalla para la guerra y, tal vez, para eventos subsecuentes, quizás nos dirá cuántos combatientes murieron y cuántos heridos hubo, cuáles comandantes se desempeñaron notablemente bien y cuáles notablemente mal, quizás nos dirá también de qué manera el tiempo y el terreno contribuyeron al resultado, tal vez incluso nos diga algo sobre la insistencia de un general en ducharse todas las mañanas a pesar de las circunstancias, o sobre el heroísmo del muchacho de los tambores, etcétera; sin embargo, probablemente nada diga sobre cuántos caballos fueron asesinados o cuántos tanques fueron destruidos y, seguramente, tampoco dirá cuántas hormigas y flores fueron aplastadas durante la batalla, etc. La crónica transmite sólo parte de toda la verdad sobre «lo que realmente pasó». Pero esto no significa que ninguna crónica histórica pueda ser (en lo que dice) verdadera; tampoco, por supuesto, que los historiadores deberían renunciar a la aspiración de descubrir verdades acerca del pasado. Dicho esto, sin embargo, se debe añadir que la incompletitud de una crónica que es verdadera pero incompleta puede ser engañosa a pesar de sus verdades. Mencionar sólo a las víctimas del campo de batalla, por ejemplo, puede inducir a error no prestando atención a los efectos de las enfermedades o al hambre sufrida por el ancho de la población, etcétera.

Las pruebas incompletas pueden inducir a error, es decir, soportar una conclusión cuya falsedad puede ser mostrada por pruebas ulteriores. Los escritores de novelas policiacas entienden esto muy bien; como cuando Spenser, el detective privado lacónico de R. B. PARKER, le dice a un testigo que le cuestiona por qué le pregunta eso: «Si yo supiera qué sería importante saber y qué no sería, tendría esta cuestión casi resuelta» ${ }^{35}$.

34 Contra F. H. BRADLEY, quien pensaba que nada menos que toda la verdad acerca de absolutamente todo era realmente-y-ciertamente verdadero. F. H. BRADLEY, Appearance and Reality: A Metaphysical Essay, Oxford, Clarendon Press, 1895, 320-321.

35 R. B. Parker, Walking Shadow (1994), New York, Penguin Putnam, 1995, 100. 
Los epistemólogos dan este aspecto por sentado: las «paradojas de GETTIER» en las que estuvieron preocupados por años, explotan el potencial para inducir al error de pruebas incompletas (al describir sus casos hipotéticos en los que alguien tiene una creencia verdadera justificada y, no obstante, no conoce, E. GETTIER da por sentado que una creencia puede estar justificada por pruebas incompletas, aun cuando de hecho puedan inducir a error y, pese a esto, ser verdadera por otras razones) ${ }^{36}$. Los especialistas en inteligencia militar a veces lo reconocen: la observación de D. RUMSFELD, muy ridiculizada pero verdadera, respecto a que al valorar la fiabilidad de la información de inteligencia sobre Irak, el gobierno estadounidense tuvo que lidiar no sólo con los elementos conocidos, no sólo con los desconocidos, sino además con los «desconocidos desconocidos», implícitamente reconoce, por ejemplo, el potencial de un conjunto de pruebas incompleto para inducir al error.

No obstante, para una auténtica precisión poética, acudo al novelista J. LENT:

La verdad no [es] una línea de aquí a allá, no se parece a los círculos que se van ampliando como los anillos de un tronco talado, sino más bien a los rastros de líquidos oscilantes entremezclados que al ser derramados asumieron una forma y vida propia, que giraron alrededor haciendo espirales y fluctuaciones para tocar y colorear todas las verdades que después emergieron de aquella ${ }^{37}$.

Precisamente, como la metáfora de la mezcla de LENT sugiere, nuevas pruebas pueden presentar algo nuevo sobre cosas que pensábamos que conocíamos: «Oh, él no estaba siendo distante porque estaba molesto conmigo», podemos decir cuando nos enteramos que su hijo está seriamente enfermo, «él está preocupado por el pequeño Johnnie, pobrecito»; o «vaya, quizá Vioxx no es un gran avance para el tratamiento de la artritis como se publicita» cuando nos enteramos que tres casos de efectos cardiovasculares adversos fueron omitidos en el informe publicado del primer importante estudio clínico hecho por Merck, el estudio VIGOR ${ }^{38}$.

Y ahora veo que, después de todo, es posible unir a la expresión idiomática «out of true», antes mencionada pero puesta a un lado, con el hilo principal de la discusión. «Out of true», como mencioné, significa «sesgado» o «torcido»; y, el resultado de decir sólo parte de la verdad, como vemos, puede ser «sesgar» o «torcer» la percepción de

36 E. GetTIER, «Is Justified True Belief Knowledge?», Analysis, 23, 1963, 121-123. Vid. también: S. HaAcK, «"Know" is Just a Four-Letter Word» (1983), en la segunda edición de Evidence and Inquiry (cita 9).

37 «The truth [is] not a line from here to there, and not ever-widening circles like the rings on a sawn log, but rather trails of oscillating overlapping liquids that poured forth but then assumed a shape and life of their own, that circled back around in spirals and fluctuations to touch and color all truths that came out after that one». J. LENT, After the Fall, New York, Vintage, 2000, 253-254.

38 C. BOMBADIER et al., «Comparison of Upper Gastrointestinal Toxicity of Rofecoxib and Naproxen in Patients With Rheumatoid Arthritis», New England Journal of Medicine, 343.21, 25 de noviembre de 2000, 1520-1528. El público se enteró en el 2005 por el estudio VIGOR, el primer estudio clínico de grandes dimensiones llevado a cabo por Merck sobre el medicamento Vioxx, que se había dado mucho más seguimiento a sus efectos gastrointestinales (que se presumían favorables de este medicamento frente a otros) que a sus efectos cardiovasculares (que se suponían desfavorables para dicha droga); de modo que los efectos cardiovasculares secundarios ocurridos durante el estudio, que una vez finalizado no tenían seguimiento, no se incluyeron en el informe publicado. D. ARMSTRONG, «How the New England Journal Missed Warning Signs on Vioxx: Medical Weekly Waited Years to Report Flaws en Article that Praised Pain Drug», Wall Street Journal, 15 de mayo de 2006, A.1, A.10. Hablo sobre esto con cierto detalle en «The Integrity of Science: What It Means, Why It Matters», en Ética e Investigacão nas Ciências da Vida - Actos do 10. Seminario do Conselho Nacional de Etica para as Ciências da Vida, 2006, 9-28; reimpreso en Putting Philosophy to Work (cita 9), 103-127. 
la audiencia sobre una verdad más amplia que no se dice. Sin duda, es también porque la verdad parcial es apta para inducir a error, «parcial» no sólo significa «incompleto», como en el segundo sentido de «verdad parcial», sino también «tendencioso» (como en «nadie en el comité fue imparcial — todos estaban más preocupados por ocultar la malversación del gobierno/de la empresa/de la universidad que en llegar al fondo del problema—, o «ese no fue un jurado imparcial —el crimen fue tan atroz que todos ellos estaban muy propensos a condenar desde el inicio por débiles que las pruebas fuesen») ${ }^{39}$. Y quizás la metáfora de LENT nos ayude a ver cómo la palabra inglesa «colorable» ha llegado a tener sus dos significados cercanos pero incompatibles: «aparentemente válido o genuino» $\mathrm{y}$ «dirigido a engañar, a falsificar» ${ }^{40}$.

Alguien puede presentarnos una verdad parcial — diciéndonos algo verdadero sólo en parte o diciéndonos menos que toda la verdad - sin intención de hacerlo o, bien, deliberadamente. Usted puede inocentemente decirme algo que es verdadero sólo en parte creyendo falsamente que todo lo dicho es verdadero; usted puede inocentemente no decir toda la verdad relevante al responder a una pregunta porque no se percata que esto o aquello es relevante (después de todo, si $p$ es relevante para $q$ depende de cuestiones fácticas y, si usted ignora o está equivocado en algunos de esos hechos, puede inocentemente omitir parte de la verdad). Y la vaguedad, de forma similar, puede ser producto de la pereza o puede ser involuntaria o puede ser deliberada.

La vaguedad deliberada puede ser benévola e incluso indispensable: según la naturaleza del caso, las normas de una Constitución, por ejemplo, deben ser lo suficientemente flexibles para servir de guía en futuras circunstancias imprevisibles en el momento en que ésta es escrita ${ }^{41}$. Por supuesto, a veces las normas jurídicas que tienen deliberadamente una textura abierta se vuelven demasiado fácilmente manipulables como para ser tan útiles como esperábamos: por ejemplo, con los criterios jurídicos para determinar si las pruebas periciales científicas ofrecidas son fiables ${ }^{42}$, o si un determinado tipo de acción gubernamental constituye una imposición religiosa ${ }^{43}$.

39 Debo advertir que, en inglés, «partial» tiene dos significados: incompleto y sesgado o tendencioso.

40 Webster's Ninth New Collegiate Dictionary, Springfield, MA, Merriam Webster, 1991.

41 Vid. también S. HAACK, «On Legal Pragmatism: Where Does "The Path of the Law" Lead Us?», American Journal of Jurisprudence, 50, 2005, 71-105, reimpreso en Pragmatism Today (próximamente); S. HAACK, «On Logic in the Law: Something, but not All», Ratio Juris, 20.1, 2007, 1-31.

${ }^{42}$ Por ejemplo el criterio Frye es más amplio si se interpreta en forma restrictiva el área de conocimiento relevante en la que un principio científico novel debe ser generalmente aceptado y, en cambio, es más limitado cuando dicha área es interpretada de forma no-restrictiva. Por ejemplo, la prueba científica novel excluida en el caso Frye (cita 18) tenía que ver con los resultados de un antiguo polígrafo al que el acusado se había sometido y había superado, mismo que la Corte sostuvo no era generalmente aceptado por psicólogos y lingüistas, la Corte confinó «comunidad relevante» a una clase mucho más pequeña de analistas del polígrafo, sin embargo, la conclusión debió ser diferente. En Daubert (cita 18), la Corte Suprema estadounidense ofreció una lista flexible de factores que los tribunales debían considerar para la admisión de pruebas periciales científicas confiables, pero también se mostró que esto puede ser manipulable. Por ejemplo, estas pruebas han sido consideradas admisibles porque están basadas en trabajos que han sido previamente sujetos a una revisión entre pares; pero también se admiten a pesar de no haber sido previamente revisados y, además, también son excluidos pese a haber sido previamente revisados. Vid., para más detalles, S. HAACK, «Peer Review and Publication: Lessons for Lawyers», Stetson Law Review, 36.7, 2007, 789-819.

43 El caso Lemon vs. Kurztman, 403 U.S. 602 (1971) era acerca de la Establishment Clause (unas de las cláusulas de la primera enmienda constitucional de los Estados Unidos sobre el Estado y la Iglesia; cláusula que establece que el gobierno no debe promover ninguna religión en particular ni la religión en general). El test presentado en este caso era que un estatuto es compatible con esta Cláusula si: i) tiene un propósito 
Y, algunas veces, el objetivo que está detrás de la vaguedad deliberada no es benévolo; busca evadir situaciones vergonzosas o inducir al error a una audiencia.

Al igual que la vaguedad deliberada, la verdad parcial deliberada es algunas veces benévola tanto en intento como en efecto. Si una amiga me pregunta qué pienso sobre su vestido nuevo y -omitiendo con mucho tacto mencionar que el estilo es feo- respondo que es un color muy bonito, pudiera estar diciendo sólo parte de la verdad esperando no herir sus sentimientos. Cuando un médico enfatiza los casos en los que cierto tratamiento ha sido exitoso, restando importancia a su alto porcentaje de ineficacia y a sus horribles efectos secundarios, podría estar diciendo sólo parte de la verdad con la esperanza de persuadir al paciente para que tome su única oportunidad, manteniéndolo lo suficientemente optimista para que no desista y renuncie.

El magistrado DARLING, a quien cité al inicio de este artículo, estaba reflexionando sobre la debilidad del testimonio de los testigos legos (su opinión de los peritos, por cierto, era que son aún menos fiables). No obstante, en el sistema jurídico estadounidense adversarial se prevé que cada uno de los litigantes acentúen la parte de verdad que conviene a sus intereses en el caso y que los abogados planteen las preguntas a sus testigos de forma concreta para que éstos puedan decir toda la verdad relevante con respecto a estas preguntas específicas, sin que se filtre ningún dato incriminatorio. Se prevé que la parte de verdad que uno de los litigantes omite será sacada a la luz por la otra parte mediante la presentación de contra-testigos o con el interrogatorio cruzado o mediante ambas; lo cual, en mi opinión, es parte de la justificación epistemológica de un sistema adversarial ${ }^{44}$ (algunas veces funciona como se espera ${ }^{45}$; pero muchas veces, me temo, no lo hace $\left.{ }^{46}\right)$.

legislativo secular; ii) su efecto primario no es difundir ni inhibir la religión, y iii) no fomenta un enredo excesivo con la religión. En decisiones subsecuentes, el inciso sobre el «enredo» se convirtió en una especie de Catch-22 [N. del T. la famosa novela de Joseph Heller] toda vez que las acciones del Estado destinadas a garantizar que un estatuto no fomentara la religión los llevo a ellos a hacer un «enredo excesivo». El actual entendimiento del segundo inciso del test Lemon — propuesto por primera vez por la magistrada O'Connor en su voto particular a favor en el caso Lynch vs. Donnelly, 465 U.S. 668 (1984), y adoptado por mayoría en su decisión County of Allegheny vs. ACLU, 492 U.S. 573 (1989) - requiere que el estatuto no transmita a un observador objetivo que el gobierno respalda o desaprueba la religión. Este «test de respaldo» puede hacerse de forma más fuerte o más débil, obviamente, dependiendo de qué exactamente el «observador objetivo» hipotético asume saber.

${ }_{44}$ El célebre estudioso de la prueba jurídica, J. WIGMORE, describió genialmente al interrogatorio cruzado como «el más grandioso motor jurídico inventado para el descubrimiento de la verdad». J. H. Wigmore, Evidence, Boston, Little, Brown \& Co., 1904, $\$ 1367$, «Cross-Examination as a Distinctive and Vital Feature of Our Law».

${ }_{45}$ See, e. g., Blum vs. Merrell Dow Pharmaceutical, 1 Pa. D. \& C. $4^{\text {th }} 634$ (1998) donde, evidentemente, el interrogatorio cruzado que hizo el abogado de Blum reveló que el consenso en la literatura científica previamente sometida a revisión entre pares, respecto a que Bendectin no era nocivo, fue artificialmente creado por la misma compañía. El caso (que fue finalmente decidido en el 2002 por la Corte Suprema de Pensilvania en favor de Merrell Dow) es discutido con mayor detalle en mi trabajo «Peer Review and Publication: Lessons for Lawyers» (cita 42).

46 Vid., por ejemplo, Barefoot vs. Estelle, 803 U.S. 880 (1983). El señor Barefoot fue condenado a pena de muerte después de que dos psiquiatras testificaron (como es requerido por el sistema de Texas en casos de pena de muerte) sobre su futura peligrosidad. La Corte Suprema estadounidense determinó que los derechos constitucionales del señor Barefoot no fueron violados. Aunque la Asociación Estadounidense de Psiquiatría envió un escrito reconociendo que las predicciones psiquiátricas sobre la futura peligrosidad de una persona están equivocadas dos de tres veces, el magistrado White en su criterio suscrito por la mayoría, dejo esto de lado señalando que los abogados del señor Barefoot tuvieron la oportunidad de someter a interrogatorio cruzado a los testigos de la fiscalía y también ofrecer testimonios de descargo. Vid. M. FrAnKEL, «The Search for Truth: An 
Pero ahora no quiero concentrarme en el caso concreto del proceso adversarial, sino en casos más sencillos en los que alguien deliberadamente no dice toda la verdad con la intención de inducir a error a su audiencia, para su propio beneficio y el perjuicio de ésta. O tal vez tendría que decir «deliberadamente o cuasi-deliberadamente» porque con mucha frecuencia lo que sucede puede ser mejor descrito como, si bien no del todo intencional, sí voluntario. Como C. NEWMAN observó: «No está en la naturaleza humana engañar a otros por mucho tiempo sin, en cierta medida, engañarnos a nosotros mismos» ${ }^{47}$. Cuando decir sólo la parte agradable o favorable de la verdad se vuelve un hábito, uno se puede encontrar fácilmente a sí mismo olvidando convenientemente los hechos que ha omitido y comenzando a creer que esta verdad parcial es toda la verdad relevante de dicha cuestión. La distinción entre alguien que sin intención induce a error diciendo sólo parte de la verdad y quien lo hace deliberadamente, es suficientemente clara en principio; pero, en la práctica, las cosas no están nada cerca de ser tan claras (lo mismo se aplica a la distinción que antes di por sentada entre alguien que sin intención está siendo vago y quien lo está siendo de forma deliberada. Mientras que en principio la diferencia es suficientemente clara, en la vida real la línea de demarcación con frecuencia es difícil de trazar; la auto-confusión conveniente no es probablemente menos ubicua que el conveniente olvido progresivo de los aspectos desagradables de la verdad. Se podría decir, haciendo eco de NEwMAN: no está en la naturaleza humana confundir a los otros por mucho tiempo sin, en cierta medida, confundirse también uno mismo).

Incluso cuando alguien dice sólo parte de la verdad creyendo inocentemente que esa es toda la verdad de la cuestión, algunas veces podemos sentir que debió haber dicho más; por supuesto no es que él debió, pues es imposible, decirnos los hechos relevantes que no conocía, pero hubiese sido deseable que agregara que podría no conocer o bien todo lo relevante o incluso qué hechos eran relevantes. Incluso cuando alguien deliberadamente o cuasi-deliberadamente dice sólo parte de la verdad por amabilidad o por tacto, algunas veces podemos sentir que hubiera sido mejor si hubiese sido más claro: quizás, por ejemplo, que yo hubiese sido una mejor amiga si con tacto hubiese sugerido que aun cuando el vestido tenía un color muy bonito, era un tanto ajustado para la entrevista de trabajo en la que planeaba usarlo; o, tal vez el médico hubiera sido más benévolo si no hubiese creado falsas esperanzas pero hubiese ayudado al paciente a aceptar lo inevitable.

Ocultar deliberadamente o cuasi-deliberadamente parte de la verdad por razones benignas es análogo a decir una «white lie» («mentira piadosa»). Ocultar deliberadamente o cuasi-deliberadamente parte de la verdad pretendiendo inducir a error a la audiencia en beneficio propio es, en mi opinión, análogo a una «black lie» («mentira no piadosa»). «Análogo a» pero no «lo mismo que» —en mi opinión, este aspecto proporciona el inicio de una explicación de por qué es tan fuerte la tentación de inducir a otros al error no diciéndoles toda la verdad y, al mismo tiempo, persuadirse uno mismo de que esta verdad es toda la verdad de la cuestión-. Las razones para engañar

\footnotetext{
Umpireal View», University of Pennsylvania Law Review, 123.5, 1975, 1031-1059; S. HAACK, «Epistemology Legalized: Or, Truth, Justice, and the American Way», American Journal of Jurisprudence, 49, 2004, $43-61$.

47 J. H. Newman, Parochial Sermons, New York, D. Appleton and Co., 1843, vol. I, sermon X, «Profession Without Practice».
} 
a otros, desde el mero interés personal hasta no estar dispuesto a ser portador de malas noticias, son suficientemente comunes. Pero decir menos que toda la verdad es con bastante frecuencia una manera especialmente atractiva de engañar a otros; es psicológicamente más fácil porque es moralmente percibido como menos grave que mentir. Si usted me dice sólo parte de la verdad (relevante), usted puede decirse a sí mismo que no me ha mentido realmente. Usted puede inducirme al error; y si omite parte de la verdad con la intención de inducirme al error, usted es culpable de engañar — pero no por mentir como tal- Y Y tendemos a sentir menos remordimiento por haber inducido al error a otros usando estas formas más sutiles y aparentemente defendibles que mintiendo abiertamente. El departamento de filosofía que revela sólo parte de la verdad sobre su éxito en encontrar empleo para sus estudiantes que han terminado el doctorado, por ejemplo, puede sentirse absuelto con la idea de que se ha sido muy cuidadoso para asegurar que todo en el prospecto de sus graduados sea verdadero ${ }^{48}$.

En sólo una semana mientras escribía este trabajo, dos artículos del Wall Street Journal cautivaron mi atención: uno decía que las compañías farmacéuticas no tienen ninguna obligación de publicar todos los estudios que realizan y presentan ante la Food and Drug Administration (Agencia de Alimentos y Medicamentos en Estados Unidos), e informaba que «se ha exagerado la efectividad de una docena de antidepresivos muy conocidos mediante la publicación selectiva de resultados favorables» ${ }^{49}$ y que esto ha llevado a muchos médicos a tomar decisiones inadecuadas sobre cómo y a qué pacientes debería prescribir tales medicamentos; el otro, advirtiendo que «con mucha frecuencia, los directivos cubren con caramelo la verdad. Eso es más peligroso que nunca», citaba a J. M. KILTS, ex-director general de Nabisco «muchas veces es lo no dicho... lo que pone a un director en problemas» ${ }^{50}$. Claramente, el engaño deliberado o cuasi-deliberado mediante una verdad incompleta parece estar absolutamente en todos lados —es mucho más frecuente, sospecho, que la mentira directa- en los negocios, en la publicidad, en la política y, siento mucho decirlo, también en la academia. Esta, sin embargo, es una historia ${ }^{51}$ para otra ocasión ${ }^{52}$.

(Traducción de Andrés Santacoloma Santacoloma y Carmen Vázquez Rojas)

48 Esta es una versión depurada de una historia verdadera que desafortunadamente ocurrió cerca de casa.

49 D. ARmSTRONG y K. J. WinSTEIN, «Antidepressants Under Scrutiny over Efficacy: Sweeping Overview Suggests Suppression of Negative Data Has Distorted View of Drugs», Wall Street Journal, 17 de enero de 2008, D.1, D.3. La cita es de p. D.1.

50 K. Whitehouse, «Why CEOs Need To Be Honest With Their Boards», Wall Street Journal, 14 de enero de 2008, R.1, R.3. Las citas son de la p. R1.

51 Vid. S. HAACK: «Out of Step: Academic Ethics in a Preposterous Environment», Putting Philosophy to Work, edición ampliada, próximamente 2012.

52 Mis agradecimientos para M. MigOTTI, por sus importantes comentarios en el borrador, a H. WOHL de la Biblioteca de Derecho de la Universidad de Miami, por ayudarme a encontrar material relevante y a M. ${ }^{a}$-J. FRÁPOLLI, por la conferencia titulada «Nothing but the Truth» que dio en 2007 en el Coloquio Nacional en Filosofía del Lenguaje en San Leopoldo, Brasil, de donde tomé la idea para el título de este trabajo. 